\title{
The Boyd-Wong idea extended
}

\section{Lech Pasicki*}

\section{"Correspondence:}

pasicki@agh.edu.pl

Faculty of Applied Mathematics,

AGH University of Science and

Technology, Al. Mickiewicza 30,

Kraków, 30-059, Poland

\begin{abstract}
Boyd and Wong in their celebrated paper 'On nonlinear contractions' assumed the comparison function to be upper semicontinuous from the right. Our requirement presented in this paper is much more general and it extends also the well-known Matkowski condition.
\end{abstract}

MSC: $47 \mathrm{H} 10 ; 54 \mathrm{H} 25$

Keywords: generalized contraction; cyclic mapping; dislocated metric; fixed point

\section{Introduction}

Boyd and Wong in [1] considered the condition $\rho(f(x), f(y)) \leq \varphi(\rho(x, y))$, where $\varphi$ : $[0, \infty) \rightarrow[0, \infty)$ is a mapping such that $\varphi(\alpha)<\alpha, \alpha>0$, and

$$
\limsup _{\beta \rightarrow \alpha^{+}} \varphi(\beta) \leq \varphi(\alpha), \quad \alpha>0,
$$

holds (i.e. $\varphi$ is upper semicontinuous from the right on $(0, \infty)$ ).

A part of [2], Theorem 3.3 shows that (1) can be replaced by

$$
\limsup _{\beta \rightarrow \alpha^{+}} \varphi(\beta)<\alpha, \quad \alpha>0 .
$$

In the present paper we apply the following condition:

$$
\text { for each } \alpha>0, \quad \varphi(\cdot) \leq \alpha \text { on some interval }(\alpha, \alpha+\epsilon) \text {. }
$$

Clearly, (3) is more general than (2). In turn, Matkowski in [3], Theorem 1.2 assumed $\varphi$ to be nondecreasing and $\lim _{n \rightarrow \infty} \varphi^{n}(\alpha)=0, \alpha>0$. It is well known that for every function $\varphi$ satisfying Matkowski's condition we have $\varphi(\alpha)<\alpha, \alpha>0$. Let us show that (3) extends the Matkowski condition for $\varphi$ such that $\varphi(\alpha)<\alpha, \alpha>0$. Assume $\varphi$ is nondecreasing, $\varphi(\beta)<\beta$, $\beta>0$, and suppose $\varphi(\cdot)>\alpha>0$ on an interval $(\alpha, \alpha+\epsilon)$. Then for any $\beta \in(\alpha, \alpha+\epsilon)$ we have $\alpha<\varphi(\beta)<\beta<\alpha+\epsilon$, and consequently, $\alpha<\varphi^{n}(\beta)<\cdots<\varphi(\beta)<\alpha+\epsilon$, i.e. $\lim _{n \rightarrow \infty} \varphi^{n}(\beta) \geq$ $\alpha>0$, a contradiction. Therefore $\varphi$ must be equal to $\alpha$ on $(\alpha, \alpha+\epsilon)$.

It is clear that (3) is equivalent to the following condition:

$$
\begin{aligned}
& \text { for each } \alpha>0 \text {, if } \limsup _{\beta \rightarrow \alpha^{+}} \varphi(\beta)=\alpha, \\
& \text { then } \varphi(\cdot) \leq \alpha \text { on some interval }(\alpha, \alpha+\epsilon),
\end{aligned}
$$

as $\lim \sup _{\beta \rightarrow \alpha^{+}} \varphi(\beta)<\alpha$ yields $\varphi(\cdot)<\alpha$ on some interval $(\alpha, \alpha+\epsilon)$.

(c) 2016 Pasicki. This article is distributed under the terms of the Creative Commons Attribution 4.0 International License (http://creativecommons.org/licenses/by/4.0/), which permits unrestricted use, distribution, and reproduction in any medium, provided you give appropriate credit to the original author(s) and the source, provide a link to the Creative Commons license, and indicate if changes were made. 


\section{Definitions and auxiliary results}

It is nice if for $f: X \rightarrow X$ the inequality

$$
\rho\left(f^{n+2}(x), f^{n+1}(x)\right) \leq \varphi\left(\rho\left(f^{n+1}(x), f^{n}(x)\right)\right), \quad n \in \mathbb{N},
$$

yields $\lim _{n \rightarrow \infty} \rho\left(f^{n+1}(x), f^{n}(x)\right)=0$. Therefore, we are interested in mappings $\varphi:[0, \infty) \rightarrow$ $[0, \infty)$ such that for each sequence $\left(a_{n}\right)_{n \in \mathbb{N}}$ the condition $0<a_{n+1} \leq \varphi\left(a_{n}\right), n \in \mathbb{N}$ yields $\lim _{n \rightarrow \infty} a_{n}=0$. The family of all such mappings was denoted in [2] by $\Psi_{\mathbf{P}}$, while the family of all mappings $\varphi:[0, \infty) \rightarrow[0, \infty)$ such that $\varphi(\alpha)<\alpha, \alpha>0$ was denoted by $\boldsymbol{\Phi}$.

Let us notice that the assumption $\varphi \in \Phi$ (or a stronger one) is present in all theorems concerning conditions (1) or (2).

\section{Proposition 2.1 $\Psi_{P} \subset \Phi$.}

Proof Suppose $\alpha \leq \varphi(\alpha)$ for a $\varphi \in \Psi_{P}$ and an $\alpha>0$. Then all $a_{n}=\alpha, n \in \mathbb{N}$ satisfy $0<a_{n+1} \leq$ $\varphi\left(a_{n}\right)$, and $\lim _{n \rightarrow \infty} a_{n}=\alpha>0$, a contradiction.

Lemma 2.2 If $a \varphi \in \Psi_{P}$, then $\varphi \in \Phi$ and (3) is satisfied.

Proof Suppose a $\varphi \in \Psi_{P}$ does not satisfy (3), i.e. there exists a sequence $\left(x_{n}\right)_{n \in \mathbb{N}}$ decreasing to an $\alpha>0$, and such that $\varphi\left(x_{n}\right)>\alpha, n \in \mathbb{N}$. Let us adopt $a_{1}=x_{1}$. There exists an $a_{2} \in\left\{x_{1}, \ldots\right\}$ such that $a_{2} \leq \varphi\left(a_{1}\right)<a_{1}$. If $a_{n}$ is defined, then $a_{n+1} \in\left\{x_{1}, \ldots\right\}$ is such that $a_{n+1} \leq \varphi\left(a_{n}\right)<a_{n}$. Our sequence $\left(a_{n}\right)_{n \in \mathbb{N}}$ satisfies $0<\alpha<a_{n+1} \leq \varphi\left(a_{n}\right), n \in \mathbb{N}$, and it does not converge to zero. Therefore, $\varphi \notin \Psi_{P}$, a contradiction.

Lemma 2.3 If a $\varphi \in \Phi$ satisfies (3), then $\varphi \in \Psi_{P}$.

Proof Let $\left(a_{n}\right)_{n \in \mathbb{N}}$ be a sequence such that $0<a_{n+1} \leq \varphi\left(a_{n}\right), n \in \mathbb{N}$ for a $\varphi \in \Phi$. Then we have

$$
0<a_{n+1} \leq \varphi\left(a_{n}\right)<a_{n}, \quad n \in \mathbb{N} .
$$

Therefore, $\left(a_{n}\right)_{n \in \mathbb{N}}$ decreases, say to an $\alpha$. Suppose $\alpha>0$. Then from (3) it follows that there exists an interval $(\alpha, \alpha+\epsilon)$ on which $\varphi(\cdot) \leq \alpha$. For large $n$ all $a_{n}$ belong to this interval. Now, we have $\alpha<a_{n+1} \leq \varphi\left(a_{n}\right) \leq \alpha$, a contradiction. Consequently, $\alpha=0$, i.e. $\varphi \in \Psi_{P}$.

Corollary 2.4 $\Psi_{P}$ consists of all mappings $\varphi \in \Phi$ satisfying (3).

Hitzler and Seda in [4] introduced the following notion of dislocated metric space.

Let $X$ be a nonempty set, and $p: X \times X \rightarrow[0, \infty)$ a mapping satisfying

$$
\begin{aligned}
& p(x, y)=0 \text { yields } x=y, \quad x, y \in X, \\
& p(x, y)=p(y, x), \quad x, y \in X, \\
& p(x, z) \leq p(x, y)+p(y, z), \quad x, y, z \in X .
\end{aligned}
$$

Then $p$ is called a dislocated metric (briefly a d-metric), and $(X, p)$ is called a dislocated metric space (briefly a d-metric space). 
If $(X, p)$ is a dislocated metric space then (see [2], (2.4))

$$
\operatorname{Ker} p=\{x \in X: p(x, x)=0\} .
$$

Let us recall that a d-metric space $(X, p)$ is called 0 -complete (see [2], Definition 2.3) if the following condition is satisfied:

$$
\begin{aligned}
& \text { for every sequence }\left(x_{n}\right)_{n \in \mathbb{N}} \text { in } X \text { such that } \lim _{m, n \rightarrow \infty} p\left(x_{n}, x_{m}\right)=0 \\
& \text { there exists an } x \in X \text { such that } \lim _{n \rightarrow \infty} p\left(x, x_{n}\right)=0 .
\end{aligned}
$$

The first idea of cyclic mappings is due to Kirk, Srinivasan and Veeramani [5]. The subsequent definition refines [2], Definition 3.6 in such a way that the case of $X=X_{1}$ is included.

Definition 2.5 A mapping $f: X \rightarrow X$ is called cyclic on $X_{1}, \ldots, X_{t}$ (for a $t \geq 1$ ) if $\emptyset \neq X=$ $X_{1} \cup \cdots \cup X_{t}$, and $f\left(X_{j}\right) \subset X_{j++}, j=1, \ldots, t$, where $j++=j+1$ for $j<t$, and $t++=1$.

Our fixed point theorems concern mappings $f: X \rightarrow X$ satisfying

$$
p(f(y), f(x)) \leq \varphi(p(y, x))
$$

or

$$
p(f(y), f(x)) \leq \varphi\left(m_{f}(y, x)\right)
$$

for

$$
m_{f}(y, x)=\max \{p(y, x), p(f(y), y), p(f(x), x)\},
$$

where $(X, p)$ is a d-metric space.

\section{Theorems}

The theorems of the present section look like some theorems from [2], but condition (3) matters a lot. Our first theorem extends [2], Theorem 3.3.

Theorem 3.1 Let $(X, p)$ be a 0-complete d-metric space, and let $f: X \rightarrow X$ be a mapping satisfying condition (6) or (7), for all $x, y \in X$ and $a \varphi \in \Phi$ such that (3) holds. Then $f$ has a unique fixed point; if $x=f(x)$, then $\lim _{n \rightarrow \infty} p\left(x, f^{n}\left(x_{0}\right)\right)=p(x, x)=0$ (i.e. $\left.x \in \operatorname{Ker} p\right), x_{0} \in X$.

Proof It is sufficient to prove that $\lim _{m, n \rightarrow \infty} p\left(x_{n}, x_{m}\right)=0$ holds for $x_{n}=f^{n}\left(x_{0}\right), n \in \mathbb{N}$ (see [2], Lemma 3.2). From the fact that $\varphi \in \Psi_{P}\left(\right.$ Lemma 2.3) it follows that $\lim _{n \rightarrow \infty} p\left(x_{n+1}, x_{n}\right)=$ 0 (see [2], Lemma 3.1). Suppose that there exists an infinite set $K \subset \mathbb{N}$ such that for each $k \in K$ there exists an $n \in \mathbb{N}$ for which $p\left(x_{n+1+k}, x_{k}\right)>\alpha>0$ holds. Let $n=n(k)>0$ be the smallest numbers satisfying this inequality for $k \in K$. For simplicity let us adopt $x=f^{k}\left(x_{0}\right)$ $\left(x_{-1}=f^{k-1}\left(x_{0}\right)\right)$, and $x_{m}=f^{m}(x), m \in \mathbb{N}$. From

$$
p\left(x_{n+1}, x\right) \leq \varphi\left(m_{f}\left(x_{n}, x_{-1}\right)\right)=\varphi\left(\max \left\{p\left(x_{n}, x_{-1}\right), p\left(x_{n+1}, x_{n}\right), p\left(x, x_{-1}\right)\right\}\right)
$$


(see (7)) we get $p\left(x_{n+1}, x\right) \leq \varphi\left(p\left(x_{n}, x_{-1}\right)\right)$, for large $k$ (or from (6) directly), as

$$
\alpha<p\left(x_{n+1}, x\right) \leq p\left(x_{n+1}, x_{n}\right)+p\left(x_{n}, x_{-1}\right)+p\left(x_{-1}, x\right) .
$$

The inequality

$$
p\left(x_{n}, x_{-1}\right) \leq p\left(x_{n}, x\right)+p\left(x, x_{-1}\right) \leq \alpha+p\left(x, x_{-1}\right)
$$

yields $p\left(x_{n}, x_{-1}\right)<\alpha+\epsilon$, for large $k$. Consequently, from (3) and $\varphi(\beta)<\alpha, \beta \leq \alpha$, we obtain

$$
\alpha<p\left(x_{n+1}, x\right) \leq \varphi\left(p\left(x_{n}, x_{-1}\right)\right) \leq \alpha,
$$

for large $k$, a contradiction, i.e. $\lim _{m, n \rightarrow \infty} p\left(x_{n}, x_{m}\right)=0$.

Now, Theorem 3.1, and [6], Lemma 29 yield the following extension of [2], Theorem 3.5.

Theorem 3.2 Let $(X, p)$ be a 0-complete $d$-metric space, and let $f: X \rightarrow X$ be a mapping satisfying condition (6) or (7), for all $x, y \in X$ with $f$ replaced by $f^{s}$ for an $s \in \mathbb{N}$, and $a \varphi \in \Phi$ having property (3). Then $f$ has a unique fixed point; if $x=f(x)$, then $\lim _{n \rightarrow \infty} p\left(x, f^{n}\left(x_{0}\right)\right)=$ $p(x, x)=0, x_{0} \in X$.

A refinement of the proof of Theorem 3.1, yields the following extension of [2], Theorem 3.9.

Theorem 3.3 Let $(X, p)$ be a 0-complete d-metric space, and let $f: X \rightarrow X$ be cyclic on $X_{1}, \ldots, X_{t}$. Assume that (6) or (7) is satisfied for all $x \in X_{j}, y \in X_{j++}, j=1, \ldots$, t and $a \varphi \in \Phi$ having property (3). Then $f$ has a unique fixed point; if $x=f(x)$, then $\lim _{n \rightarrow \infty} p\left(x, f^{n}\left(x_{0}\right)\right)=$ $p(x, x)=0, x_{0} \in X$.

Proof It is sufficient to prove that $\lim _{m, n \rightarrow \infty} p\left(x_{n}, x_{m}\right)=0$ holds for $x_{n}=f^{n}\left(x_{0}\right), n \in \mathbb{N}$ (see [2], Lemma 3.8). From the fact that $\varphi \in \Psi_{P}$ (Lemma 2.3) it follows that $\lim _{n \rightarrow \infty} p\left(x_{n+1}, x_{n}\right)=$ 0 (see [2], Lemma 3.7). Suppose that there exists an infinite set $K \subset \mathbb{N}$ such that for each $k \in K$ there exists an $n \in \mathbb{N}$ for which $p\left(x_{(n+1) t+k+1}, x_{k}\right)>\alpha>0$ holds. Let $n=n(k)>0$ be the smallest numbers satisfying this inequality for $k \in K$. For simplicity let us adopt $x=f^{k}\left(x_{0}\right)$ $\left(x_{-1}=f^{k-1}\left(x_{0}\right)\right)$, and $x_{m}=f^{m}(x), m \in \mathbb{N}$. Clearly, $x \in X_{j}$ yields $x_{n t+1}, x_{(n+1) t+1} \in X_{j++}$. In view of (7) we have

$$
\begin{aligned}
p\left(x_{(n+1) t+1}, x\right) & \leq \varphi\left(m_{f}\left(x_{(n+1) t}, x_{-1}\right)\right) \\
& =\varphi\left(\max \left\{p\left(x_{(n+1) t}, x_{-1}\right), p\left(x_{(n+1) t+1}, x_{(n+1) t}\right), p\left(x, x_{-1}\right)\right\}\right),
\end{aligned}
$$

which, for large $k$ (or from (6) directly) gives

$$
p\left(x_{(n+1) t+1}, x\right) \leq \varphi\left(p\left(x_{(n+1) t}, x_{-1}\right)\right)
$$

as

$$
\alpha<p\left(x_{(n+1) t+1}, x\right) \leq p\left(x_{(n+1) t+1}, x_{(n+1) t}\right)+p\left(x_{(n+1) t}, x_{-1}\right)+p\left(x_{-1}, x\right) .
$$


Now,

$$
\begin{aligned}
p\left(x_{(n+1) t}, x_{-1}\right) & \leq p\left(x_{(n+1) t}, x_{(n+1) t-1}\right)+\cdots+p\left(x_{n t+2}, x_{n t+1}\right)+p\left(x_{n t+1}, x\right)+p\left(x, x_{-1}\right) \\
& \leq p\left(x_{(n+1) t}, x_{(n+1) t-1}\right)+\cdots+p\left(x_{n t+2}, x_{n t+1}\right)+\alpha+p\left(x, x_{-1}\right)
\end{aligned}
$$

yields $p\left(x_{(n+1) t}, x_{-1}\right)<\alpha+\epsilon$, for large $k$. Consequently, from (3) and $\varphi(\beta)<\alpha, \beta \leq \alpha$, we obtain

$$
\alpha<p\left(x_{(n+1) t+1}, x\right) \leq \varphi\left(p\left(x_{(n+1) t}, x_{-1}\right)\right) \leq \alpha,
$$

for large $k$, a contradiction. Now, it is clear that $\lim _{m, n \rightarrow \infty} p\left(x_{m+n t+1}, x_{m}\right)=0$. One step more is necessary for $t>1$. We have

$$
\begin{aligned}
& \lim _{m, n \rightarrow \infty} p\left(x_{m+n t+s}, x_{m}\right) \\
& \quad \leq \lim _{m, n \rightarrow \infty}\left[p\left(x_{m+n t+s}, x_{m+n t+s-1}\right)+\cdots+p\left(x_{m+n t+2}, x_{m+n t+1}\right)+p\left(x_{m+n t+1}, x_{m}\right)\right]=0
\end{aligned}
$$

for any $s \in\{2, \ldots, t\}$, i.e. $\lim _{m, n \rightarrow \infty} p\left(x_{n}, x_{m}\right)=0$.

Clearly Theorem 3.3 is more general than Theorem 3.1. The proof of Theorem 3.1 is easier, it helps to understand the idea of the proof of Theorem 3.3, and therefore, it is also presented.

Now, Theorem 3.3, and [6], Lemma 29 yield the following.

Theorem 3.4 Let $(X, p)$ be a 0 -complete d-metric space, and let $f: X \rightarrow X$ be a mapping such that $f^{s}$ is cyclic on $X_{1}, \ldots, X_{t}$ for an $s \in \mathbb{N}$. Assume that (6) or (7) is satisfied for all $x \in X_{j}, y \in X_{j_{+}}, j=1, \ldots, t$ with $f$ replaced by $f^{s}$, and $a \varphi \in \Phi$ having property (3). Then $f$ has a unique fixed point; if $x=f(x)$, then $\lim _{n \rightarrow \infty} p\left(x, f^{n}\left(x_{0}\right)\right)=p(x, x)=0, x_{0} \in X$.

Remark 3.5 Let us note that [2], Lemmas 3.2, 3.8 stay valid if we assume that $(X, p)$ is 0 -complete for orbits of $f$, i.e. (5) holds for $x_{n}=f^{n}\left(x_{0}\right), x_{m}=f^{m}\left(x_{0}\right), m, n \in \mathbb{N}, x_{0} \in X$. Consequently, theorems of Section 3 stay valid if the assumption that $(X, p)$ is 0 -complete is replaced by the requirement that $(X, p)$ is 0 -complete for orbits of $f$.

Competing interests

The author declares that he has no competing interests.

\section{Acknowledgements}

The work has been supported by the Polish Ministry of Science and Higher Education.

Received: 10 March 2016 Accepted: 6 May 2016 Published online: 21 May 2016

\section{References}

1. Boyd, DW, Wong, JSW: On nonlinear contractions. Proc. Am. Math. Soc. 20, 458-464 (1969)

2. Pasicki, L: Dislocated metric and fixed point theorems. Fixed Point Theory Appl. 2015, 82 (2015)

3. Matkowski, J: Integrable solutions of functional equations. Diss. Math. 127, 1-68 (1975)

4. Hitzler, P, Seda, AK: Dislocated topologies. J. Electr. Eng. 51(12), 3-7 (2000)

5. Kirk, WA, Srinivasan, PS, Veeramani, P: Fixed points for mappings satisfying cyclical contractive conditions. Fixed Point Theory 4, 79-89 (2003)

6. Pasicki, L: Fixed point theorems for contracting mappings in partial metric spaces. Fixed Point Theory Appl. 2014, 185 (2014) 